\title{
GROUPS OF NONLINEAR CONGRUENCES AND GEODESIC LINES ON THE ORDERED SET OF POSITIVE DEFINITE MATRICES
}

\section{F. Bessmertnyi}

\begin{abstract}
A family of nonlinear transformation groups of Hermitian matrices preserving the basic linear congruences properties is constructed. Each group generates a structure of a partially ordered vector multispace on the set of Hermitian matrices. Determinant inequalities which allow us to find geodesic lines for an analog of indefinite metric on positive definite matrix set are obtained.
\end{abstract}

Mathematics Subject Classification (2010). 15B48; 20F29; 53F30.

Key words: nonlinear congruences, Lorentz nonlinear transformations, partial orders, indefinite metric, triangle reverse inequality, geodesic lines, determinant inequalities, majorization.

- $\mathbb{H}_{n}$ stands for the set of all Hermitian $n \times n$ matrices with complex entries.

- $\mathbb{P}_{n}$ stands for the subset of all positive semidefinite $n \times n$ matrices.

- $\mathbb{P}_{n}^{+}$stands for the subset of all positive definite $n \times n$ matrices.

- $G L(n, \mathbb{C})$ stands for the group of all invertible $n \times n$ matrices with respect to the ordinary multiplication of matrices.

- $S L(n, \mathbb{C})$ stands for the group of $n \times n$ matrices with a unit determinant.

- $U(n)$ stands for the group of unitary $n \times n$ matrices.

- $S U(n)$ stands for the group of unitary $n \times n$ matrices with a unit determinant.

\section{Introduction}

Invariance and causality are fundamental principles of physics. Coordinate transformations play an important role, if they preserve some notion of positivity. This explains a special interest of mathematicians and physicists in a partially ordered set of positive definite matrices. The congruence transformation $X^{\prime}=Q \cdot X \cdot Q^{*}$, where $Q \in G L(n, \mathbb{C})$ and $Q^{*}$ is Hermitian conjugate matrix, is the linear transformation of $\mathbb{H}_{n}$. The congruences preserve positive definiteness and the standard partial order relation $X \leq Y$ (in the sense of quadratic forms). Riemannian metrics on the set of positive definite matrices and automorphisms of corresponding structures are intensively studied (see [1]-[4], [13, chapter 6]).

In this article we introduce the simplest nonlinear transformations of $\mathbb{H}_{n}$. These transformations preserve the ordinary congruence basic properties. The nonlinear congruent transformations define the structure of so-called ordered vector multispace on $\mathbb{H}_{n}$. They are order automorphisms for this structure. In addition, these transformations define an invariant scalar function on a pair of positive definite matrices (an analogue of the point-dependent indefinite metric). The triangle inverse inequality is performed on the causally connected point triple. If a pair of points is causally connected, then there is a structure of a vector space on $\mathbb{H}_{n}$ such that the equation of geodesic (of maximum "length") is a linear equation. The geodesic line point determines the addition operation choice in this equation. It's addition operation is the operation of vector multispace.

\section{Groups of non-linear congruences}

2.1. Recall that inertia of $X \in \mathbb{H}_{n}$ is the triple of nonnegative integers

$$
\operatorname{In}(X)=\{\pi(X), \varsigma(X), v(X)\},
$$


where $\pi(X), \varsigma(X), v(X)$ are the numbers of positive, zero, and negative eigenvalues of $X$ (counted with multiplicity). The transformation

$$
Y=l_{Q}(X)=Q \cdot X \cdot Q^{*}, Q \in G L(n, \mathbb{C})
$$

of $\mathbb{H}_{n}$ onto itself is called a congruence.

We list the characteristic properties of congruences:

(i). The set of all congruences (2.1) form a arcwise connected linear transformation group of $\mathbb{H}_{n}$ with respect to the superposition of mappings;

(ii). $\operatorname{In}\left(l_{Q}(X)\right)=\operatorname{In}(X)$ for all $X \in \mathbb{H}_{n}$. In particular $l_{Q}(X) \geq 0 \Leftrightarrow X \geq 0$;

(iii). If $Q \in S L(n, \mathbb{C})$, then $\operatorname{det} l_{Q}(X)=\operatorname{det} X$ for all $X \in \mathbb{H}_{n}$.

2.2. Suppose $F$ is an arbitrary one-to-one mapping of $\mathbb{H}_{n}$ onto itself, $F^{-1}$ is the reverse mapping for $F$, and $f \circ g$ is the superposition of mappings. Let $l_{Q}$ be congruence, i.e. $l_{Q}(X)=Q \cdot X \cdot Q^{*}$, and $X \in \mathbb{H}_{n}$. The set of all mappings

$$
\varphi_{Q}(X)=F \circ l_{Q} \circ F^{-1}(X), Q \in G L(n, \mathbb{C})
$$

is a group of transformations of $\mathbb{H}_{n}$ with respect to the superposition of mappings. This group is denoted by $G_{F}(n)$.

We will to call the group $G_{F}(n)$ is regular if $\operatorname{In} \varphi_{Q}(X) \equiv \operatorname{In} X$ for all $Q \in G L(n, \mathbb{C})$ and $\operatorname{det} \varphi_{Q}(X) \equiv \operatorname{det} X$ for all $Q \in S L(n, \mathbb{C})$.

2.3 Lemma. The group $G_{F}(n)$ is regular if and only if

$$
\text { In } X_{1}=\operatorname{In} X_{2}, \operatorname{det} X_{1}=\operatorname{det} X_{2} \text { implies In } F\left(X_{1}\right)=\operatorname{In} F\left(X_{2}\right), \operatorname{det} F\left(X_{1}\right)=\operatorname{det} F\left(X_{2}\right) .
$$

Proof. Let $G_{F}(n)$ be regular. For any $X_{1}, X_{2}, \operatorname{In} X_{1}=\operatorname{In} X_{2}, \operatorname{det} X_{1}=\operatorname{det} X_{2}$ there exists a congruence $l_{Q}$ such that $X_{2}=l_{Q}\left(X_{1}\right), Q \in S L(n, \mathbb{C})$. The mapping $X_{2}^{\prime}=\varphi_{Q}\left(X_{1}^{\prime}\right)$ closes the commutative diagram

$$
\begin{array}{rlrl}
X_{1} \in \mathbb{H}_{n} \quad \stackrel{l_{Q}}{\longrightarrow} \quad \begin{aligned}
X_{2} & \in \mathbb{H}_{n} \\
& F \downarrow
\end{aligned} F^{-1} \uparrow & & \\
X_{1}^{\prime} \in \mathbb{H}_{n} \quad \stackrel{\varphi_{Q}}{\longrightarrow} \quad X_{2}^{\prime} \in \mathbb{H}_{n}
\end{array}
$$

Since group $G_{F}(n)$ is regular and $Q \in S L(n, \mathbb{C})$, then $\operatorname{In} F\left(X_{1}\right)=\operatorname{In} F\left(X_{2}\right), \operatorname{det} F\left(X_{1}\right)=\operatorname{det} F\left(X_{2}\right)$. Sufficiency is obvious.

Let $f$ be one-to-one mapping of $\mathbb{R}$ onto itself. For any matrix $X \in \mathbb{H}_{n}$ with spectral value decomposition $X=U \cdot \Lambda \cdot U^{*}$, where $\Lambda=\operatorname{diag}\left\{\lambda_{1}, \cdots, \lambda_{n}\right\}$ and $U$ is unitary, we define

$$
f(X)=U \cdot \operatorname{diag}\left\{f\left(\lambda_{1}\right), \cdots, f\left(\lambda_{n}\right)\right\} \cdot U^{*} .
$$

It is clear that $Y=f(X)$ is one-to-one mapping of $\mathbb{H}_{n}$ onto itself.

2.4 Theorem. Let $f$ be one-to-one mapping of $\mathbb{R}$ onto itself, continuous over all of $\mathbb{R}$, and $f(-1)=-f(1)$. The group $G_{f}(n)$ is regular if and only if

$$
f(x) \equiv C_{0} \cdot|x|^{\delta} \cdot \operatorname{sgn} x, \delta>0, C_{0} \neq 0 .
$$


Proof. Let $G_{f}(n)$ be regular. Since $f$ is one-to-one on $\mathbb{R}$ and $f(-1)=-f(1)$, then $f(1)=C_{0} \neq 0$. The function $h(x)=f(x) / f(1)$ satisfies the condition (2.3) and $h(1)=1$. Choosing $X_{1}=\operatorname{diag}\{-x, 1,1, \cdots, 1\}, X_{2}=\operatorname{diag}\{x,-1,1, \cdots, 1\}$, we get $\operatorname{det} h\left(X_{1}\right)=\operatorname{det} h\left(X_{2}\right)$, i.e. $h(-x)=-h(x)$.

Let $X_{1}=\operatorname{diag}\{\lambda \mu, 1,1, \cdots, 1\}, X_{2}=\operatorname{diag}\{\lambda, \mu, 1, \cdots, 1\}$, where $\lambda, \mu>0$. By Lemma 2.3 , we have

$$
h(\lambda \mu)=h(\lambda) h(\mu), \lambda, \mu>0 .
$$

In the class of continuous functions on the positive semi-axis the solutions of Cauchy functional equation (2.6) are functions $h(x)=x^{\alpha}, \alpha \in \mathbb{R}$ and only they. By condition $h(x)$ is continuous at point 0 and one-to-one on $\mathbb{R}$. Then $\alpha>0$. Using condition $h(-x)=-h(x)$ we obtain (2.5). Sufficiency is obvious.

Now we shall give the following definition.

2.5 Definition. Let $\tau_{\delta}(x)=|x|^{\delta} \cdot \operatorname{sgn} x, \delta>0 \quad$ and $\quad \tau_{\delta}^{-1}(y)=|y|^{1 / \delta} \cdot \operatorname{sgn} y$. The set of all transformations

$$
X^{\prime}=\varphi_{Q}(X)=\tau_{\delta}\left[Q \cdot \tau_{\delta}^{-1}(X) \cdot Q^{*}\right], Q \in G L(n, \mathbb{C}), \quad X, X^{\prime} \in \mathbb{H}_{n},
$$

is called the group of $\delta$-congruences and denote by $G_{\delta}(n)$. If $Q \in S L(n, \mathbb{C})$, then the mapping (2.7) is called unimodular $\delta$-congruence. The subgroup of unimodular $\delta$-congruences denote by $S G_{\delta}(n)$.

2.6 Remark. If $\delta=1$, then group $G_{\delta}(n)$ is a group of ordinary congruences.

2.7. The following results are easily checked directly from the definition:

(i). $Q, R \in G L(n, \mathbb{C}) \Rightarrow \varphi_{Q} \circ \varphi_{R}=\varphi_{Q \cdot R}, \quad \varphi_{Q}^{-1}=\varphi_{Q^{-1}}$;

(ii). $Q \in G L(n, \mathbb{C}) \Rightarrow \operatorname{In} \varphi_{Q}(X)=\operatorname{In} X$ for all $X \in \mathbb{H}_{n}$. In particular $\varphi_{Q}(X) \geq 0 \Leftrightarrow X \geq 0$;

(iii). $Q \in S L(n, \mathbb{C}) \Rightarrow \operatorname{det} \varphi_{Q}(X)=\operatorname{det} X$ for all $X \in \mathbb{H}_{n}$;

(iv). If $\alpha \in \mathbb{R}$, then $\varphi_{Q}(\alpha \cdot X)=\alpha \cdot \varphi_{Q}(X)$.

Proof (iv): If $\alpha \in \mathbb{R}$, then $\varphi_{Q}(\alpha \cdot X)=\tau_{\delta}\left[Q \cdot \tau_{\delta}^{-1}(\alpha \cdot X) \cdot Q^{*}\right]=\tau_{\delta} \circ \tau_{\delta}^{-1}(\alpha) \cdot \varphi_{Q}(X)=\alpha \cdot \varphi_{Q}(X)$

For any matrix $Q \in G L(n, \mathbb{C})$ with singular value decomposition $Q=U \cdot \Lambda \cdot V$ (see [11], p. 150), where $U, V$ is unitary and $\Lambda=\operatorname{diag}\left\{\alpha_{1}, \cdots, \alpha_{n}\right\}>0$, we have (see 2.7 (i))

$$
\varphi_{Q}=\varphi_{U \cdot \Lambda \cdot V}=\varphi_{U} \circ \varphi_{\Lambda} \circ \varphi_{V}
$$

Next we will need the following properties of mappings $\varphi_{Q} \in G_{\delta}(n)$.

2.8 Proposition. The mappings $\varphi_{Q}$ of group $G_{\delta}(n)$ satisfy the conditions:

(i). If $W \in U(n)$, then $\varphi_{W}(X) \equiv W X W^{*}$;

(ii). If $\delta \neq 1$ and $\Lambda=\operatorname{diag}\left\{\alpha_{1}, \cdots, \alpha_{n}\right\}>0$, then $\varphi_{\Lambda}(X+Y)=\varphi_{\Lambda}(X)+\varphi_{\Lambda}(Y)$ for all $X, Y \in \mathbb{H}_{n}$ if and only if $\alpha_{1}=\alpha_{2}=\cdots=\alpha_{n}$;

(iii). If $0 \neq \varsigma \in \mathbb{C}$, then $\varphi_{\varsigma \cdot Q}(X)=|\varsigma|^{2 \delta} \cdot \varphi_{Q}(X)$ for all $X \in \mathbb{H}_{n}$;

(iv). If $\delta \neq 1$, then $\varphi_{Q}(X+Y)=\varphi_{Q}(X)+\varphi_{Q}(Y)$ for all $X, Y \in \mathbb{H}_{n}$ if and only if $\varsigma \cdot Q \in U(n)$ for some complex number $\varsigma \neq 0$;

(v). Let $\operatorname{In} Y=\operatorname{In} X$. There exists $\varphi_{Q} \in G_{\delta}(n)$ such that $Y=\varphi_{Q}(X)$. If moreover $\operatorname{det} Y=\operatorname{det} X$, then $\varphi_{Q} \in S G_{\delta}(n)$. 
Proof (i) - (v):

(i). By definition (2.4), we have $\varphi_{W}(X)=\tau_{\delta}\left[W \tau_{\delta}^{-1}(X) W^{*}\right]=W\left[\tau_{\delta} \circ \tau_{\delta}^{-1}(X)\right] W^{*}=W X W^{*}$;

(ii). The general case is reduced to a case of $2 \times 2$ matrices. Let

$$
X=\left(\begin{array}{ll}
1 & 0 \\
0 & 1
\end{array}\right), \quad Y=\left(\begin{array}{ll}
0 & 1 \\
1 & 0
\end{array}\right), \quad \Lambda=\left(\begin{array}{cc}
\alpha_{1} & 0 \\
0 & \alpha_{2}
\end{array}\right)>0
$$

Then

$$
\begin{gathered}
\varphi_{\Lambda}(X)+\varphi_{\Lambda}(Y)=\left(\begin{array}{cc}
\alpha_{1}^{2 \delta} & 0 \\
0 & \alpha_{2}^{2 \delta}
\end{array}\right)+\left(\begin{array}{cc}
0 & \alpha_{1}^{\delta} \alpha_{2}^{\delta} \\
\alpha_{1}^{\delta} \alpha_{2}^{\delta} & 0
\end{array}\right)=\left(\begin{array}{cc}
\alpha_{1}^{2 \delta} & \alpha_{1}^{\delta} \alpha_{2}^{\delta} \\
\alpha_{1}^{\delta} \alpha_{2}^{\delta} & \alpha_{2}^{2 \delta}
\end{array}\right) ; \\
\varphi_{\Lambda}(X+Y)=\varphi_{\Lambda}\left(\begin{array}{ll}
1 & 1 \\
1 & 1
\end{array}\right)=2^{1-\delta}\left(\alpha_{1}^{2}+\alpha_{2}^{2}\right)^{\delta-1} \cdot\left(\begin{array}{cc}
\alpha_{1}^{2} & \alpha_{1} \alpha_{2} \\
\alpha_{1} \alpha_{2} & \alpha_{2}^{2}
\end{array}\right) .
\end{gathered}
$$

Comparing (2.9) and (2.10), we obtain $2^{1-\delta}\left(\alpha_{1}^{2}+\alpha_{2}^{2}\right)^{\delta-1}=\alpha_{i}^{\delta-1} \alpha_{j}^{\delta-1}, i, j=1,2$. If $\delta \neq 1$, then $\alpha_{1}=\alpha_{2}$. Sufficiency is obvious;

(iii). We have

$$
\varphi_{\varsigma \cdot Q}(X) \stackrel{\text { def }}{=} \tau_{\delta}\left[\varsigma Q \cdot \tau_{\delta}^{-1}(X) \cdot \bar{\varsigma} Q^{*}\right]=\tau_{\delta}\left(|\varsigma|^{2}\right) \cdot \tau_{\delta}\left[Q \cdot \tau_{\delta}^{-1}(X) \cdot Q^{*}\right]=|\varsigma|^{2 \delta} \cdot \varphi_{Q}(X)
$$

(iv) follows from (i), (ii), (iii) and (2.8);

(v). Let $X, Y \in \mathbb{H}_{n}$ and $\operatorname{In} X=\operatorname{In} Y$. There are exists $U, V \in U(n)$ such that $X=U \cdot \Lambda_{X} \cdot U^{*}$, $Y=V \cdot \Lambda_{Y} \cdot V^{*}$, where

$$
\Lambda_{X}=\operatorname{diag}\left\{\lambda_{1}, \cdots, \lambda_{n}\right\}, \Lambda_{Y}=\operatorname{diag}\left\{\mu_{1}, \cdots, \mu_{n}\right\} \quad \text { and } \operatorname{sgn} \lambda_{k}=\operatorname{sgn} \mu_{k}, k=1, \cdots, n .
$$

By definition, put

$$
Q=V \cdot \Delta \cdot U^{*}, \Delta=\operatorname{diag}\left\{\alpha_{1}, \cdots, \alpha_{n}\right\}, \text { where } \alpha_{k}=\left|\mu_{k} / \lambda_{k}\right|^{1 / 2 \delta} \text { if } \lambda_{k} \neq 0, \text { otherwise } \alpha_{k}=1 .
$$

Then

$$
\varphi_{Q}(X)=\tau_{\delta}\left[V \cdot \Delta \cdot U^{*} \cdot \tau_{\delta}^{-1}(X) \cdot U \cdot \Delta \cdot V^{*}\right]=V \cdot \tau_{\delta}\left[\Delta \cdot \tau_{\delta}^{-1}\left(\Lambda_{X}\right) \cdot \Delta\right] \cdot V^{*}=V \cdot \tau_{\delta}\left[\tau_{\delta}^{-1}\left(\Lambda_{Y}\right)\right] \cdot V^{*}=Y .
$$

If $\operatorname{det} Y=\operatorname{det} X$, then, as is easy to see, the determinant of $\Delta$ can be chosen equal to 1

\section{Vector Multispace}

First we consider the question of a suitable definition of a vector multispace.

3.1 Proposition. Let $L$ be a vector space with the operations $x+y, \alpha \cdot x$. Each one-to-one mapping $\varphi$ of the set L onto itself defines on L new operations $\underset{\oplus}{\oplus} y, \alpha \times \underset{\varphi}{\varphi}$ such that:

(i). The set $L$ with operations $x \stackrel{\varphi}{\oplus} y, \underset{\alpha}{\oplus} \times x$ is a new vector space $L_{\varphi}$;

(ii). The mapping $\varphi: L \rightarrow L_{\varphi}$ is a linear operator.

Proof. By definition, put

$$
x \stackrel{\varphi}{\oplus} y=\varphi\left[\varphi^{-1}(x)+\varphi^{-1}(y)\right], \quad \stackrel{\varphi}{\alpha \times x}=\varphi\left[\alpha \cdot \varphi^{-1}(x)\right] .
$$

The set $L$ is new vector space with operations (3.1). Moreover:

$$
\varphi(x+y)=\varphi\left[\varphi^{-1}(\varphi(x))+\varphi^{-1}(\varphi(y))\right]=\varphi(x) \stackrel{\varphi}{\oplus} \varphi(y) ; \quad \varphi(\alpha \cdot x)=\varphi\left[\alpha \cdot \varphi^{-1}(\varphi(x))\right]=\stackrel{\varphi}{\alpha \times \varphi(x)}
$$


Let $G$ be a group of nonlinear transformations of the vector space $L$.

The vector multispace of pair $\{L, G\}$ is the space $L$ with the set operations (3.1), where $\varphi$ runs over all group $G$.

Remark. Each mapping $\varphi \in G$ is automorphism of vector multispace of pair $\{L, G\}$ :

$$
\begin{gathered}
\varphi(x \oplus y)=\varphi \circ \psi\left[\psi^{-1} \circ \varphi^{-1}(\varphi(x))+\psi^{-1} \circ \varphi^{-1}(\varphi(y))\right]=\varphi(x) \stackrel{\varphi \circ \psi}{\oplus} \varphi(y) ; \\
\varphi(\alpha \times x)=\varphi \circ \psi\left[\alpha \cdot \psi^{-1} \circ \varphi^{-1}(\varphi(x))\right]=\alpha \circ \varphi(x) .
\end{gathered}
$$

The set $\mathbb{H}_{n}$ is $n^{2}$ - dimensional vector space over $\mathbb{R}$ with respect to the ordinary addition of matrices and multiplication matrix by a number. Let $G=G_{\delta}(n)$ be a group of $\delta$-congruences.

3.2 Definition. The $\delta$-multispace $\mathbb{H}_{\delta}(n)$ is the vector multispace of pair $\left\{\mathbb{H}_{n}, G_{\delta}(n)\right\}$. $\square$.

Now proceed on to research the operations of multispace $\mathbb{H}_{\delta}(n)$.

Symbols $\underset{\alpha \times}{\alpha} X, X \stackrel{Q}{\oplus} Y$ by denote the operations (3.1) corresponding to the mapping $\varphi_{Q} \in G_{\delta}(n)$. From the homogeneity of transformations $\varphi_{Q}$ (see 2.7 (iv)) we obtain

3.3 Proposition. There exists a unique multiplication operation by a number in $\mathbb{H}_{\delta}(n)$.

Proof. By definition $\underset{\alpha}{\alpha} X=\varphi_{Q}\left[\alpha \cdot \varphi_{Q}^{-1}(X)\right]=\alpha \cdot \varphi_{Q}\left[\varphi_{Q}^{-1}(X)\right]=\alpha \cdot X$

3.4 Proposition. The addition's operations of multispace $\mathbb{H}_{\delta}(n)$ satisfy the following conditions:

(i). If $Q, R \in G L(n, \mathbb{C})$, then $\varphi_{R}(X \stackrel{Q}{\oplus} Y)=\varphi_{R}(X) \stackrel{R \cdot Q}{\oplus} \varphi_{R}(Y)$ for all $X, Y \in \mathbb{H}_{\delta}(n)$;

(ii). If $W \in U(n)$, then $X \stackrel{W}{\oplus} Y=X+Y$ for all $X, Y \in \mathbb{H}_{\delta}(n)$;

(iii). If $Y=\beta \cdot X$, then $X \stackrel{Q}{\oplus} Y=X+Y$ for all $Q \in G L(n, \mathbb{C})$;

(iv). If $Q \in G L(n, \mathbb{C})$ and $0 \neq \varsigma \in \mathbb{C}$, then $X \stackrel{\varsigma \cdot Q}{\oplus} Y=X \stackrel{Q}{\oplus} Y$ for all $X, Y \in \mathbb{H}_{\delta}(n)$;

(v). If $X, Y \geq 0$, then $X \stackrel{Q}{\oplus} Y \geq 0$ for all $Q \in G L(n, \mathbb{C})$.

Proof (i) - (v):

(i) follows from (3.2);

(ii). By Proposition 2.8 (i) $X \stackrel{W}{\oplus} Y=\varphi_{W}\left[\varphi_{W}^{-1}(X)+\varphi_{W}^{-1}(Y)\right]=W\left[W^{*} X W+W^{*} Y W\right] W^{*}=X+Y$;

(iii). We have $X \stackrel{Q}{\oplus} Y=X \stackrel{Q}{\oplus}(\beta \cdot X)=(1+\beta) \cdot X=X+\beta \cdot X=X+Y$;

(iv). Using Propositions 2.7 (iv) and 2.8 (iii), we get

$$
X \stackrel{\varsigma \cdot Q}{\oplus} Y=\varphi_{\varsigma \cdot Q}\left[\varphi_{\varsigma \cdot Q}^{-1}(X)+\varphi_{\zeta \cdot Q}^{-1}(Y)\right]=|\varsigma|^{2 \delta} \cdot \varphi_{Q}\left[|\varsigma|^{-2 \delta} \cdot \varphi_{Q}^{-1}(X)+|\varsigma|^{-2 \delta} \cdot \varphi_{Q}^{-1}(Y)\right]=X \stackrel{Q}{\oplus} Y ;
$$

(v). Since the mappings $\varphi_{Q}$ and $\varphi_{Q}^{-1}$ preserve inertia, then $X \stackrel{Q}{\oplus} Y=\varphi_{Q}\left[\varphi_{Q}^{-1}(X)+\varphi_{Q}^{-1}(Y)\right] \geq 0 . \mathbf{\square}$.

From Proposition 3.4 (iv) we obtain

3.5 Corollary. Let $S G_{\delta}(n)$ is a group of unimodular $\delta$-congruences (Definition 2.5). The multispaces of pairs $\left\{\mathbb{H}_{n}, S G_{\delta}(n)\right\}$ and $\left\{\mathbb{H}_{n}, G_{\delta}(n)\right\}$ are the same.

Let $Q=|Q|_{L} \cdot W$ is polar value decomposition for $Q \in G L(n, \mathbb{C})$, where $|Q|_{L}=\left(Q \cdot Q^{*}\right)^{1 / 2}>0$ uniquely determined and $W \in U(n)$. 
3.6 Proposition. Let $\delta>0$. If $Q \in G L(n, \mathbb{C})$, then $X \stackrel{Q}{\oplus} Y=X \stackrel{||_{L}}{\oplus} Y$ for all $X, Y \in \mathbb{H}_{\delta}(n)$.

Proof. If $Q=|Q|_{L} \cdot W$, then

$$
\begin{aligned}
& X \stackrel{Q}{\oplus} Y=\varphi_{Q}\left[\varphi_{Q}^{-1}(X)+\varphi_{Q}^{-1}(Y)\right]=\varphi_{\left.Q\right|_{L}} \circ \varphi_{W}\left[\varphi_{W}^{-1}\left(\varphi_{\left.Q\right|_{L}}^{-1}(X)\right)+\varphi_{W}^{-1}\left(\varphi_{|Q|_{L}}^{-1}(Y)\right)\right]= \\
& =\left.\varphi_{Q Q}\right|_{L} W\left[W^{*} \varphi_{|Q|_{L}}^{-1}(X) W+W^{*} \varphi_{\left.Q\right|_{L}}^{-1}(Y) W\right] W^{*}=X \stackrel{|Q|_{L}}{\oplus} Y
\end{aligned}
$$

3.7 Corollary. Let $\delta \neq 1$ and $R, Q \in S L(n, \mathbb{C})$. The addition's operations $X \stackrel{R}{\oplus} Y, X \stackrel{Q}{\oplus} Y$ are the same if and only if $|R|_{L}=|Q|_{L}$.

Proof. Supposing $X \stackrel{R}{\oplus} Y=X \stackrel{Q}{\oplus} Y$ for all $X, Y \in \mathbb{H}_{n}$, we obtain

$$
\varphi_{Q}^{-1} \circ \varphi_{R}\left[\varphi_{R}^{-1}(X)+\varphi_{R}^{-1}(Y)\right]=\varphi_{Q}^{-1}(X)+\varphi_{Q}^{-1}(Y)=\varphi_{Q}^{-1} \circ \varphi_{R}\left[\varphi_{R}^{-1}(X)\right]+\varphi_{Q}^{-1} \circ \varphi_{R}\left[\varphi_{R}^{-1}(Y)\right],
$$

i.e. $\varphi_{Q}^{-1} \circ \varphi_{R}=\varphi_{Q^{-1} \cdot R}$ is linearly mapping. By Proposition 2.8 (iv) $Q^{-1} R=W \in U(n)$, i.e. $R=Q \cdot W$. Then $|R|_{L}=\left(Q \cdot W \cdot W^{*} \cdot Q^{*}\right)^{1 / 2}=\left(Q \cdot Q^{*}\right)^{1 / 2}=|Q|_{L}$.

Proposition 3.4(iv), Corollary 3.7 and Remark 2.6 mean that we have proved

3.8 Theorem. If $\delta>0, \delta \neq 1$, then exist is a one-to-one correspondence between the set of addition's operations of multispace $\mathbb{H}_{\delta}(n)$ and the set $S \mathbb{P}_{n}^{+}$of positive definite matrices with a unit determinant. If $\delta=1$, then multispace $\mathbb{H}_{1}(n)$ coincides with the vector space $\mathbb{H}_{n}$. $\mathbf{}$.

3.9 Remark. A group $S G_{\delta}(n)$ of unimodular congruences is the smallest subgroup in $G_{\delta}(n)$ that defines the same set of linear operation on $\mathbb{H}_{\delta}(n)$ that all group $G_{\delta}(n)$.

\section{Partially Ordered Vector Multispace}

4.1. A subset $C$ of a vector space $L$ is convex cone if $\alpha x+\beta y$ belongs to $C$ for any positive scalars $\alpha, \beta$, and any $x, y$ in $L$. A cone is called closed if it contains all its limit points. A cone is called pointed if $C \bigcap(-C)=\{\theta\}$.

Each closed pointed convex cone $C$ induces (see [14]) a partial ordering $\leq$ on $L$ so that $x \leq y$ if and only if $y-x \in C$. This partial ordering compatible with the vector space structure, i.e. for all $x, y, z$ in $L$ and $0<\lambda$ in $\mathbb{R}$ the following two axioms are satisfied

$$
x \leq y \text { implies } x+z \leq y+z ; \quad x \leq y \text { implies } \lambda x \leq \lambda y .
$$

A vector space with such an order is called an ordered vector space.

There exists a one-to-one correspondence between the partial orders on a vector space $L$ that are compatible with the vector space structure and the closed pointed convex cones of $L$.

4.2. Let's list the requirements for the concept of partial order on vector multispace $\mathbb{H}_{\delta}(n)$ :

(i). A relation of partial order must be compatible with all operations of multispace $\mathbb{H}_{\delta}(n)$;

(ii). A relation of partial order must be preserving when transformations of group $G_{\delta}(n)$.

4.3 Proposition. The set $\mathbb{P}_{n}$ of all positive semidefinite Hermitian $n \times n$ matrices is the unique (up to multiplication by (-1) nontrivial closed pointed convex (with respect to each addition) cone in $\mathbb{H}_{\delta}(n)$ that is invariant under all transformations of group $G_{\delta}(n)$. 
Proof. The set of all matrices of the same inertia is the smallest (with respect to the set-theoretic inclusion) subset in $\mathbb{H}_{\delta}(n)$ that invariant under the all transformations of the group $G_{\delta}(n)$. Among a nontrivial invariant subsets in $\mathbb{H}_{\delta}(n)$ (the matrices with the same nonzero inertia) only the set $\mathbb{P}_{n}^{+}$of positive definite and the set $-\mathbb{P}_{n}^{+}$of negatively definite matrices are convex (with respect to the each addition) cones. Hence closure $\mathbb{P}_{n}$ of the set $\mathbb{P}_{n}^{+}$is the desired closed pointed convex cone in $\mathbb{H}_{\delta}(n)$.

Given the requirements of $\mathbf{4 . 2}$ (i) we introduce the following definition.

4.4 Definition. The vector multispace $\mathbb{H}_{\delta}(n)$ is called the ordered vector multispace, if defined partial order $\leq_{\delta}$ on $\mathbb{H}_{\delta}(n)$ such that

$$
X \leq_{\delta} Y \quad \text { if and only if } \quad Y \stackrel{Q}{\ominus} X \in \mathbb{P}_{n} \quad \text { for all } Q \in G L(n, \mathbb{C}) .
$$

4.5 Remark. Considering Corollary 3.8, we can replace the group $G L(n, \mathbb{C})$ by the unimodular group $S L(n, \mathbb{C})$ in condition (4.1).

There are several conditions that characterize partial ordering $\leq_{\delta}$ on the set of positive definite matrices $\mathbb{P}_{n}^{+}$at different values $\delta>0$.

4.6 Theorem. Let $X, Y \in \mathbb{P}_{n}^{+}$. If $0<\delta \leq 1$, then the following conditions are equivalent:

(i). $X \leq_{\delta} Y$;

(ii). $Y \stackrel{\tau_{2 \delta}^{-1}(X)}{\ominus} X \geq 0$;

(iii). $Y^{1 / \delta} \geq X^{1 / \delta}$.

Proof. (i) $\Rightarrow$ (ii). Let $X \leq_{\delta} Y$, i.e. $\forall Q \in G L(n, \mathbb{C}): \stackrel{Q}{\ominus} X \geq 0$. Then $Y \stackrel{\tau_{2 \delta}^{-1}(X)}{\ominus} X \geq 0$.

(ii) $\Rightarrow$ (iii). If $Y \stackrel{\tau_{2 \delta}^{-1}(X)}{\Theta} X \geq 0$, then

$$
\begin{aligned}
\varphi_{X^{1 / 2}}\left[\varphi_{X^{1 / 2 \delta}}^{-1}(Y)-\varphi_{X^{1 / 2 \delta}}^{-1}(X)\right] \geq 0 \Rightarrow \varphi_{X^{1 / 2 \delta}}\left[\left(X^{-1 / 2 \delta} Y^{1 / \delta} X^{-1 / 2 \delta}\right)^{\delta}-I_{n}\right] \geq 0 \Rightarrow \\
\Rightarrow\left(X^{-1 / 2 \delta} Y^{1 / \delta} X^{-1 / 2 \delta}\right)^{\delta}-I_{n} \geq 0 \Rightarrow X^{-1 / 2 \delta} Y^{1 / \delta} X^{-1 / 2 \delta} \geq I_{n} \Rightarrow Y^{1 / \delta} \geq X^{1 / \delta} .
\end{aligned}
$$

(iii) $\Rightarrow$ (i). If $Y^{1 / \delta} \geq X^{1 / \delta}$, then $Q^{-1} Y^{1 / \delta} Q^{-1 *} \geq Q^{-1} X^{1 / \delta} Q^{-1 *}$ for all $Q \in G L(n, \mathbb{C})$. Since $0<\delta \leq 1$, then $f(X)=X^{\delta}$ is monotone on $\mathbb{P}_{n}^{+}$. Hence

$$
\left(Q^{-1} Y^{1 / \delta} Q^{-1 *}\right)^{\delta} \geq\left(Q^{-1} X^{1 / \delta} Q^{-1 *}\right)^{\delta} \Rightarrow \varphi_{Q}\left[\varphi_{Q}^{-1}(Y)-\varphi_{Q}^{-1}(X)\right] \geq 0 \Rightarrow X \leq_{\delta} Y . \boldsymbol{\square} .
$$

We need the following Lemma to characterize the partial order at $\delta>1$.

4.7 Lemma. Let $\delta>0$ and $X, Y \in \mathbb{P}_{n}^{+}$. There exists a transformation $\varphi_{Q_{0}} \in S G_{\delta}(n)$ such that

$$
\varphi_{Q_{0}}(X)=\lambda_{0} \cdot I_{n}, \quad \varphi_{Q_{0}}(Y)=\Lambda_{\mu}=\operatorname{diag}\left\{\mu_{1}, \cdots, \mu_{n}\right\},
$$

where $\lambda_{0}=\sqrt[n]{\operatorname{det} X}$ and $\operatorname{det} Y=\operatorname{det} \Lambda_{\mu}$.

Proof. Since $\operatorname{In} X=\operatorname{In}\left(\lambda_{0} I_{n}\right)$ and $\operatorname{det} X=\operatorname{det}\left(\lambda_{0} I_{n}\right)$, then (Proposition $2.8(\mathrm{v})$ ) there is exist $R \in S L(n, \mathbb{C})$ such that

$$
\varphi_{R}(X)=\lambda_{0} \cdot I_{n}, \varphi_{R}(Y)=Y^{\prime}>0 .
$$

Let $Q_{0}=W \cdot R$, where $W \cdot Y^{\prime} \cdot W^{*}=\Lambda_{\mu}=\operatorname{diag}\left\{\mu_{1}, \cdots, \mu_{n}\right\}$, and $W \in S U(n)$. Then 


$$
\varphi_{Q_{0}}(X)=\varphi_{W \cdot R}(X)=W \cdot\left(\lambda_{0} \cdot I_{n}\right) \cdot W^{*}=\lambda_{0} \cdot I_{n}, \varphi_{Q_{0}}(Y)=\varphi_{W \cdot R}(Y)=W \cdot Y^{\prime} \cdot W^{*}=\Lambda_{\mu} \cdot
$$

4.8 Theorem. Let $\delta>1$. If $X, Y \in \mathbb{P}_{n}^{+}$, then

$$
X \leq_{\delta} Y \quad \text { if and only if } Y=\beta \cdot X, \beta \geq 1 .
$$

Proof. Let $Q=Q_{0}^{-1} \cdot U_{n} \cdot \Lambda_{n}$, where $Q_{0}$ satisfies conditions of Lemma 4.7 and

$$
U_{n}=\left(\begin{array}{cc}
U_{2} & 0 \\
0 & I_{n-2}
\end{array}\right), U_{2}=\left(\begin{array}{cc}
1 / \sqrt{2} & 1 / \sqrt{2} \\
-1 / \sqrt{2} & 1 / \sqrt{2}
\end{array}\right) ; \quad \Lambda_{n}=\left(\begin{array}{cc}
\Lambda_{2} & 0 \\
0 & I_{n-2}
\end{array}\right), \Lambda_{2}=\left(\begin{array}{cc}
\alpha & 0 \\
0 & \alpha^{-1}
\end{array}\right)>0 .
$$

Since $Q=Q_{0}^{-1} \cdot U_{n} \cdot \Lambda_{n} \in S L(n, \mathbb{C})$, then

$$
\begin{gathered}
\operatorname{det}(Y \stackrel{Q}{\ominus} X)=\operatorname{det} \varphi_{Q_{0}^{-1} \cdot U_{n} \cdot \Lambda_{n}}\left[\varphi_{U_{n} \cdot \Lambda_{n}}^{-1}\left(\varphi_{Q_{0}}(Y)\right)-\varphi_{U_{n} \cdot \Lambda_{n}}^{-1}\left(\varphi_{Q_{0}}(X)\right)\right]= \\
=\operatorname{det}\left[\varphi_{\Lambda_{n}}^{-1}\left(U_{n}^{*} \cdot \Lambda_{\mu}^{(n)} \cdot U_{n}\right)-\lambda_{0} \cdot \varphi_{\Lambda_{n}}^{-1}\left(I_{n}\right)\right]=\left(\mu_{3}-\lambda_{0}\right) \cdots\left(\mu_{n}-\lambda_{0}\right) \cdot \operatorname{det}\left[\varphi_{\Lambda_{2}}^{-1}\left(U_{2}^{*} \cdot \Lambda_{\mu}^{(2)} \cdot U_{2}\right)-\lambda_{0} \cdot \varphi_{\Lambda_{2}}^{-1}\left(I_{2}\right)\right],
\end{gathered}
$$

where $\Lambda_{\mu}^{(n)}=\varphi_{Q_{0}}(Y)=\operatorname{diag}\left\{\mu_{1}, \cdots, \mu_{n}\right\}, \Lambda_{\mu}^{(2)}=\operatorname{diag}\left\{\mu_{1}, \mu_{2}\right\}, \quad \lambda_{0} \cdot I_{n}=\varphi_{Q_{0}}(X)$.

By condition $Y \stackrel{Q}{\ominus} X \geq 0$ for all $Q \in S L(n, \mathbb{C})$. Then $\mu_{k} \geq \lambda_{0}, k=1, \cdots, n$. We to prove that from conditions $X \leq_{\delta} Y$ and $\delta>1$, we obtaining $\mu_{2}=\mu_{1}$. We have

$$
\begin{aligned}
& g(\alpha)=\operatorname{det}\left[\varphi_{\Lambda_{2}}^{-1}\left(U_{2}^{*} \cdot \Lambda_{\mu}^{(2)} \cdot U_{2}\right)-\lambda_{0} \cdot \varphi_{\Lambda_{2}}^{-1}\left(I_{2}\right)\right]= \\
&=\operatorname{det}\left[2^{-\delta}\left(\begin{array}{cc}
\alpha^{-2}\left(\mu_{1}^{1 / \delta}+\mu_{2}^{1 / \delta}\right) & \left(\mu_{1}^{1 / \delta}-\mu_{2}^{1 / \delta}\right) \\
\left(\mu_{1}^{1 / \delta}-\mu_{2}^{1 / \delta}\right) & \alpha^{2}\left(\mu_{1}^{1 / \delta}+\mu_{2}^{1 / \delta}\right)
\end{array}\right)^{\delta}-\lambda_{0} \cdot\left(\begin{array}{cc}
\alpha^{-2 \delta} & 0 \\
0 & \alpha^{2 \delta}
\end{array}\right)\right] .
\end{aligned}
$$

The analysis shows that

$$
g(\alpha) \sim-\frac{\lambda_{0}}{2^{\delta}} \alpha^{-4(\delta-1)}\left(\mu_{1}^{1 / \delta}+\mu_{2}^{1 / \delta}\right)^{\delta-2}\left(\mu_{1}^{1 / \delta}-\mu_{2}^{1 / \delta}\right)^{2} \quad \text { at } \quad \alpha \rightarrow 0+.
$$

If $\mu_{1}^{1 / \delta}-\mu_{2}^{1 / \delta} \neq 0$ and $\delta>1$, then $\lim _{\alpha \rightarrow 0+} g(\alpha)=-\infty$, which contradicts the condition $Y \stackrel{Q}{\ominus} X \geq 0$. Then $\mu_{2}=\mu_{1}$. Similarly we get $\mu_{k}=\mu_{1}$ for $k=3, \cdots, n$. Hence $\Lambda_{\mu}^{(n)}=\mu_{1} \cdot I_{n}$ and

$$
Y=\varphi_{Q_{0}}^{-1}\left(\Lambda_{\mu}^{(n)}\right)=\varphi_{Q_{0}}^{-1}\left(\mu_{1} \cdot I_{n}\right)=\beta \cdot \varphi_{Q_{0}}^{-1}\left(\lambda_{0} \cdot I_{n}\right)=\beta \cdot X, \quad \beta=\mu_{1} / \lambda_{0} \geq 1 .
$$

Sufficiency is obvious.

The partial order relation $X \leq_{\delta} Y$ satisfies the condition 4.2(ii).

4.9 Proposition. Let $X, Y>0, \varphi_{Q} \in G_{\delta}(n)$. Then $X \leq_{\delta} Y$ in $\mathbb{H}_{\delta}(n)$ if and only if $\varphi_{Q}(X) \leq_{\delta} \varphi_{Q}(Y)$.

Proof. If $0<\delta \leq 1$, then

$$
X \leq_{\delta} Y \Leftrightarrow X^{1 / \delta} \leq Y^{1 / \delta} \Leftrightarrow Q \cdot X^{1 / \delta} \cdot Q^{*} \leq Q \cdot Y^{1 / \delta} \cdot Q^{*} \Leftrightarrow\left[\varphi_{Q}(X)\right]^{1 / \delta} \leq\left[\varphi_{Q}(Y)\right]^{1 / \delta} \Leftrightarrow \varphi_{Q}(X) \leq_{\delta} \varphi_{Q}(Y) .
$$

If $\delta>1$, then $X \leq_{\delta} Y \Leftrightarrow Y=\beta \cdot X, \beta \geq 1 \Leftrightarrow \varphi_{Q}(Y)=\beta \cdot \varphi_{Q}(X) \Leftrightarrow \varphi_{Q}(Y) \leq_{\delta} \varphi_{Q}(X)$

In conclusion, we present some inequalities for the $Q$ - sum of positive definite matrices. We will need the following F. Hansen's result.

Theorem (see [5]). Let $Y$ and $Q$ be bounded linear operators on Hilbert space $H$. We suppose that $Y \geq 0$ and $\|Q\| \leq 1$. If $f$ is an operator monotone function defined on $[0, \infty)$, then

$$
Q \cdot f(Y) \cdot Q^{*} \leq f\left(Q \cdot Y \cdot Q^{*}\right) .
$$


4.10 Proposition. Let $X \geq 0$ and $Q Q^{*} \leq I_{n}$. If $0<\delta_{1} \leq \delta_{2} \leq 1$, then

$$
\left(Q \cdot X^{1 / \delta_{2}} \cdot Q^{*}\right)^{\delta_{2}} \leq\left(Q \cdot X^{1 / \delta_{1}} \cdot Q^{*}\right)^{\delta_{1}} \cdot
$$

Proof. If $X \geq 0$, then $Y=X^{1 / \delta_{1}} \geq 0$. The function $f(x)=x^{\delta_{1} / \delta_{2}}$ is an operator monotone function on $[0, \infty)$. By inequality $(4.5)$ we obtain

$$
Q \cdot\left(X^{1 / \delta_{1}}\right)^{\delta_{1} / \delta_{2}} \cdot Q^{*} \leq\left(Q \cdot X^{1 / \delta_{1}} \cdot Q^{*}\right)^{\delta_{1} / \delta_{2}} \Rightarrow Q \cdot X^{1 / \delta_{2}} \cdot Q^{*} \leq\left(Q \cdot X^{1 / \delta_{1}} \cdot Q^{*}\right)^{\delta_{1} / \delta_{2}} .
$$

Since $f(x)=x^{\delta_{2}}$ is an operator monotone function on $[0, \infty)$, then (4.6) is true.

4.11 Corollary. Suppose $X \geq 0$ and $\alpha_{1} \geq \cdots \geq \alpha_{n}>0$ are singular numbers of the matrix $Q \in G L(n, \mathbb{C})$. If $0<\delta_{1} \leq \delta_{2} \leq 1$, then

$$
\left(Q \cdot X^{1 / \delta_{2}} \cdot Q^{*}\right)^{\delta_{2}} \leq \alpha_{1}^{2\left(\delta_{2}-\delta_{1}\right)} \cdot\left(Q \cdot X^{1 / \delta_{1}} \cdot Q^{*}\right)^{\delta_{1}} ; \quad\left(Q^{-1} \cdot X^{1 / \delta_{2}} \cdot Q^{-1 *}\right)^{\delta_{2}} \leq \alpha_{n}^{-2\left(\delta_{2}-\delta_{1}\right)} \cdot\left(Q^{-1} \cdot X^{1 / \delta_{1}} \cdot Q^{-1 *}\right)^{\delta_{1}} \cdot
$$

Proof. If $Q \in G L(n, \mathbb{C})$, then $Q_{1}=\alpha_{1}^{-1} Q$ satisfy the condition $Q_{1} Q_{1}^{*} \leq I_{n}$. By Proposition 4.10 we have

$$
\left(Q_{1} \cdot X^{1 / \delta_{2}} \cdot Q_{1}^{*}\right)^{\delta_{2}} \leq\left(Q_{1} \cdot X^{1 / \delta_{1}} \cdot Q_{1}^{*}\right)^{\delta_{1}} \Rightarrow \alpha_{1}^{-2 \delta_{2}} \cdot\left(Q \cdot X^{1 / \delta_{2}} \cdot Q^{*}\right)^{\delta_{2}} \leq \alpha_{1}^{-2 \delta_{1}} \cdot\left(Q \cdot X^{1 / \delta_{1}} \cdot Q^{*}\right)^{\delta_{1}} .
$$

Similarly for the matrix $Q_{2}=\alpha_{n} \cdot Q^{-1}, Q_{2} Q_{2}^{*} \leq I_{n}$.

Let $0<\delta \leq 1$ and $X, Y \geq 0$. We introduce the notations

$$
\left(Q \cdot X^{1 / \delta} \cdot Q^{*}\right)^{\delta} \stackrel{\text { def }}{=} \varphi_{\delta, Q}(X) ; \quad \varphi_{\delta, Q}\left[\varphi_{\delta, Q}^{-1}(X)+\varphi_{\delta, Q}^{-1}(Y)\right] \stackrel{\text { def }}{=} X \stackrel{Q}{\oplus} Y .
$$

4.12 Theorem. Suppose $X, Y \geq 0$ and the matrix $Q \in G L(n, \mathbb{C})$ has singular numbers $\alpha_{1} \geq \cdots \geq \alpha_{n}>0$. If $0<\delta_{1} \leq \delta_{2} \leq 1$, then

$$
X \stackrel{Q}{\oplus_{\delta_{2}}} Y \leq\left(\alpha_{1} / \alpha_{n}\right)^{2\left(\delta_{2}-\delta_{1}\right)} \cdot\left(X \stackrel{Q}{\oplus_{\delta_{1}}} Y\right) .
$$

Proof. Using Corollary 4.11 we obtain

$$
\begin{aligned}
X \stackrel{Q}{\oplus}_{\delta_{2}}^{Q} Y & =\varphi_{\delta_{2}, Q}\left[\varphi_{\delta_{2}, Q}^{-1}(X)+\varphi_{\delta_{2}, Q}^{-1}(Y)\right] \leq \alpha_{1}^{2\left(\delta_{2}-\delta_{1}\right)} \cdot \varphi_{\delta_{1}, Q}\left[\varphi_{\delta_{2}, Q^{-1}}(X)+\varphi_{\delta_{2}, Q^{-1}}(Y)\right] \leq \\
\leq & \alpha_{1}^{2\left(\delta_{2}-\delta_{1}\right)} \cdot \varphi_{\delta_{1}, Q}\left[\alpha_{n}^{-2\left(\delta_{2}-\delta_{1}\right)} \cdot \varphi_{\delta_{1}, Q^{-1}}(X)+\alpha_{n}^{-2\left(\delta_{2}-\delta_{1}\right)} \cdot \varphi_{\delta_{1}, Q^{-1}}(Y)\right]= \\
& =\left(\alpha_{1} / \alpha_{n}\right)^{2\left(\delta_{2}-\delta_{1}\right)} \cdot \varphi_{\delta_{1}, Q}\left[\varphi_{\delta_{1}, Q}^{-1}(X)+\varphi_{\delta_{1}, Q}^{-1}(Y)\right]=\left(\alpha_{1} / \alpha_{n}\right)^{2\left(\delta_{2}-\delta_{1}\right)} \cdot\left(X \stackrel{Q}{\oplus_{\delta_{1}}} Y\right) .
\end{aligned}
$$

\section{Chains in Ordered Vector Multispace $\mathbb{H}_{\delta}(n)$}

A linearly ordered subset in a partially ordered set is called a chain. Let $X_{0}$ be fixed positive definite matrix of the partially ordered vector multispace $\mathbb{H}_{\delta}(n)$. Consider the set of all chains in $\mathbb{P}_{n}^{+} \subseteq \mathbb{H}_{\delta}(n)$ passing through the point $X_{0}$.

5.1 Proposition. If $\delta>1$, then through each point $X_{0} \in \mathbb{P}_{n}^{+}$in $\mathbb{H}_{\delta}(n)$ passes a unique chain.

Proof. By Theorem 4.8 we have $X_{0} \leq_{\delta} X$ if and only if $X=\beta \cdot X_{0}, \beta \geq 1$. Therefore, there is a unique chain in $\mathbb{P}_{n}^{+}$passing through the point $X_{0}$ is determined by the equation

$$
X=s \cdot X_{0}, s>0 . \boldsymbol{\square} .
$$

5.2. Let $0<\delta \leq 1$ and $X_{0}, X_{1}$ are fixed positive definite matrices satisfying the condition $X_{0} \leq_{\delta} X_{1}$. By Definition 4.4 and Remark $4.5 \quad X \leq_{\delta} Y$ if and only if $Y \stackrel{Q}{\ominus} X \geq 0$ for all 
$Q \in S L(n, \mathbb{C})$. Denote by $S_{k}(A)$ the elementary symmetric polynomial of degree $k=1, \cdots, n$ from the eigenvalues of the matrix $A$. By the definition $Y \stackrel{Q}{\ominus} X \geq 0$ if and only if the eigenvalues of $Y \stackrel{Q}{\ominus} X$ are nonnegative numbers. It is easy to show that

$$
X_{0} \leq_{\delta} X_{1} \Leftrightarrow S_{k}\left(X_{1} \stackrel{Q}{\ominus} X_{0}\right) \geq 0, k=1, \cdots, n \text { for all } Q \in S L(n, \mathbb{C}) .
$$

The map $X^{\prime}=\varphi_{R}(X)$ of the group $S G_{\delta}(n)$ take each $S_{k}\left(X_{1} \stackrel{Q}{\ominus} X_{0}\right)$ to $S_{k}\left[\varphi_{R}\left(X_{1} \stackrel{Q}{\ominus} X_{0}\right)\right]$.

5.3 Proposition. Let $0<\delta \leq 1$ and $X_{0}, X_{1} \in \mathbb{H}_{\delta}(n)$. The number $S_{n}\left(X_{1} \stackrel{Q}{\ominus} X_{0}\right)$ is an invariant of the group $S G_{\delta}(n)$. For $k=1, \cdots, n-1$ the number $S_{k}\left(X_{1} \stackrel{Q}{\ominus} X_{0}\right)$ is an invariant of the group $S G_{\delta}(n)$ if and only if $S_{k}\left(X_{1} \stackrel{Q}{\ominus} X_{0}\right)=0$.

Proof. The invariance of $S_{n}\left(X_{1} \stackrel{Q}{\ominus} X_{0}\right)=\operatorname{det}\left(X_{1} \stackrel{Q}{\ominus} X_{0}\right)$ is obvious (see 2.7(iii)). Consider one of the numbers $S_{k}\left(X_{1} \stackrel{Q}{\ominus} X_{0}\right)$, where $k=1, \cdots, n-1$. If $S_{k}\left(X_{1} \stackrel{Q}{\ominus} X_{0}\right)=0$, then $\operatorname{rank}\left(X_{1} \stackrel{Q}{\ominus} X_{0}\right)<k$. The transformation $\varphi_{R}$ of the group $S G_{\delta}(n)$ preserve the inertia. Then $\operatorname{rank} \varphi_{R}\left(X_{1} \stackrel{Q}{\ominus} X_{0}\right)<k$, i.e. $S_{k}\left[\varphi_{R}\left(X_{1} \stackrel{Q}{\ominus} X_{0}\right)\right]=0$. If $S_{k}\left(X_{1} \stackrel{Q}{\ominus} X_{0}\right) \neq 0$, then transformations of the group $S G_{\delta}(n)$ change its value. Indeed, because the eigenvalues of $X_{1} \stackrel{Q}{\ominus} X_{0}$ do not change during transformation $\varphi_{W}(X)=W \cdot X \cdot W^{*}, W \in S U(n)$, we can assume that

$$
X_{1} \stackrel{Q}{\ominus} X_{0}=\Lambda_{\mu}=\operatorname{diag}\left\{\mu_{1}, \cdots, \mu_{n}\right\},
$$

where $\mu_{1} \cdots \mu_{k} \neq 0$. Let $R=\operatorname{diag}\left\{1, \cdots, 1, \alpha_{k}, \alpha_{k+1}, 1, \cdots, 1,\right\}, \alpha_{k} \cdot \alpha_{k+1}=1$. It is easy to see that the value of $S_{k}\left[\varphi_{R}\left(X_{1} \stackrel{Q}{\ominus} X_{0}\right)\right]$ can be made arbitrarily large by selecting the appropriate number $\alpha_{k}$..

5.4. Let $0<\delta \leq 1$ and $X_{0}, Y_{0}$ be positive definite matrices in $\mathbb{H}_{\delta}(n)$. If $X_{0} \leq_{\delta} Y_{0}$, then the points $X_{0}, Y_{0}$ can be connected by a set of chains. By Proposition 5.3 the number $s(Q)=S_{n}\left(X_{1} \stackrel{Q}{\ominus} X_{0}\right)=\operatorname{det}\left(X_{1} \stackrel{Q}{\ominus} X_{0}\right)$ is an invariant of group $S G_{\delta}(n)$ for each matrix $Q \in G L(n, \mathbb{C})$. Among all chains connecting points $X_{0}, Y_{0}$ we are interested in an extreme chain for which the function $s(Q)=\operatorname{det}\left(X_{1} \stackrel{Q}{\ominus} X_{0}\right)$ takes an extreme value. We will need some determinant inequalities.

\section{Determinant inequalities for positive definite matrices}

In this section, we prove for vector multispace $\mathbb{H}_{\delta}(n)$ the Minkowski's determinant inequality and the following theorem.

6.1 Theorem. Let $0<\delta \leq 1$ and $X, Y$ be positive defined $n \times n$-matrices. If $X \leq_{\delta} Y$, then

$$
\forall Q \in G L(n, \mathbb{C}): \operatorname{det}(Y \stackrel{Q}{\ominus} X) \geq \operatorname{det}\left(Y \stackrel{\tau_{2 \delta}^{-1}(X)}{\ominus} X\right) .
$$

The inequality holds for any pair of positive definite matrices at $n=2$.

6.2. Recall some definitions. For any $x=\left(x_{1}, x_{2}, \cdots, x_{n}\right) \in \mathbb{R}^{n}$, let $x_{\downarrow}=\left(x_{[1]}, x_{[2]}, \cdots, x_{[n]}\right)$ denote the decreasing rearrangement of $x$, i. e., $x_{[1]} \geq x_{[2]} \geq \cdots \geq x_{[n]}$. The elementwize vector ordering $x_{j} \leq y_{j}, j=1, \cdots, n$, is denoted by $x \leq y$. By definition, put $\log x=\left(\log x_{1}, \cdots, \log x_{n}\right)$. 
For $x, y \in \mathbb{R}^{n} y$ is said to be majorizes $x$ (see [10], [12]) if

$$
\sum_{i=1}^{k} x_{[i]} \leq \sum_{i=1}^{k} y_{[i]}, \quad 1 \leq k \leq n-1 \quad \text { and } \quad \sum_{i=1}^{n} x_{[i]}=\sum_{i=1}^{n} y_{[i]},
$$

this relation is denoted by $x \prec y$.

Let $X \in \mathbb{P}_{n}^{+}$. The eigenvalues of $X$ are denoted by $\lambda(X)=\left(\lambda_{1}, \lambda_{2}, \cdots, \lambda_{n}\right)_{\downarrow}$, i.e. $\lambda_{1} \geq \lambda_{2} \geq \cdots \geq \lambda_{n}>0$.

Let $\mathbb{D}_{+}^{n}(1)=\left\{x \in \mathbb{R}^{n}: x_{1} \geq 1, \cdots, x_{n} \geq 1\right\}$. For a set $\mathbb{A} \subseteq \mathbb{R}^{n}$

$$
x \prec y \text { on } \mathbb{A}
$$

means $x, y \in \mathbb{A}$ and $x \prec y$.

Proposition 6.3 is an analogue of Schur's result (see [10], p. 78) for log- majorization.

6.3 Proposition. If $\log \left(x_{1}, \cdots, x_{n}\right) \prec \log \left(y_{1}, \cdots, y_{n}\right)$ on $\mathbb{D}_{+}^{n}(1)$, then

$$
\left(x_{1}-1\right) \cdots\left(x_{n}-1\right) \geq\left(y_{1}-1\right) \cdots\left(y_{n}-1\right) \text {. }
$$

Proof. Let $\tilde{z}_{k}=\prod_{j=1}^{k} z_{j}, k=1, \cdots, n$. Then $\log x \prec \log y$ on $\mathbb{D}_{+}^{n}(1)$ if and only if

$$
x_{1} \geq \cdots \geq x_{n} \geq 1, \quad y_{1} \geq \cdots \geq y_{n} \geq 1, \quad \tilde{x}_{k} \leq \tilde{y}_{k}, k=1, \cdots, n-1 \text { and } \tilde{x}_{n}=\tilde{y}_{n} .
$$

Let $\hat{y}$ be a constant vector in $\mathbb{D}_{+}^{n}(1)$. On the set $\mathbb{D}=\left\{x \in \mathbb{D}_{+}^{n}(1): \log x \prec \log \hat{y}\right\}$ consider the function

$$
\phi\left(x_{1}, \cdots, x_{n}\right)=\left(x_{1}-1\right) \cdots\left(x_{n}-1\right)=\left(\tilde{x}_{1}-1\right)\left(\tilde{x}_{2} / \tilde{x}_{1}-1\right) \cdots\left(\tilde{x}_{n-1} / \tilde{x}_{n-2}-1\right)\left(\tilde{x}_{n} / \tilde{x}_{n-1}-1\right) .
$$

Since $\tilde{y}_{n}$ is constant and $\tilde{x}_{n}=\tilde{y}_{n}$, then $\partial \varphi / \partial \tilde{x}_{n} \equiv 0$. Therefore $\log x \prec \log y$ on $\mathbb{D}$ implies $\phi(x) \geq \phi(y)$ if and only if

$$
\phi\left(x_{1}, \cdots, x_{n}\right)=\left(\tilde{x}_{1}-1\right)\left(\tilde{x}_{2} / \tilde{x}_{1}-1\right) \cdots\left(\tilde{x}_{n-1} / \tilde{x}_{n-2}-1\right)\left(\tilde{x}_{n} / \tilde{x}_{n-1}-1\right)
$$

is decreasing in $\tilde{x}_{j}, j=1, \cdots, n-1$, over the region where $x \in \mathbb{D}$. Since

$$
\frac{\partial \varphi}{\partial \tilde{x}_{k}}=-\frac{x_{k}-x_{k+1}}{x_{1} \cdots x_{k}}\left(x_{1}-1\right) \cdots\left(x_{k-1}-1\right)\left(x_{k+2}-1\right) \cdots\left(x_{n}-1\right) \leq 0 \quad \text { on } \quad \mathbb{D}_{+}^{n}(1),
$$

then (6.3) is true.

The following two well-known theorems generalize the inequality Lieb and Tearing ([6], [7]). 6.4 Theorem (see [8], Theorem 6.ii). Let $X, Y$ are positive defined Hermitian $n \times n$ matrices. If $0<\alpha<\beta$, then

$$
\log \lambda^{1 / \alpha}\left(X^{\alpha} Y^{\alpha}\right) \prec \log \lambda^{1 / \beta}\left(X^{\beta} Y^{\beta}\right)
$$

6.5 Theorem (see [9], Theorem 2.1). Let $A, B$ are positive definite $n \times n$ matrices. If $0<\delta \leq 1$, then

$$
\operatorname{tr}\left(A^{\delta} \cdot B^{\delta} \cdot A^{\delta}\right) \leq \operatorname{tr}(A \cdot B \cdot A)^{\delta},
$$

where $\operatorname{tr} X$ stands for matrix trace.

To prove Theorem 6.1, we need several lemmas.

6.6 Lemma. If $A, B$ is $n \times n$ positive defined Hermitian matrices and $0<\alpha \leq 1$, then

$$
\log \lambda\left(A^{\alpha} B^{\alpha} A^{\alpha}\right) \prec \log \lambda(A B A)^{\alpha} .
$$

Proof. If $A>0$, then $\lambda(A B)=\lambda\left(A^{1 / 2} B A^{1 / 2}\right)$. Taking into account Theorem 6.4, we obtain

$$
\log \lambda^{1 / \alpha}\left(A^{\alpha / 2} B^{\alpha} A^{\alpha / 2}\right) \prec \log \lambda^{1 / \beta}\left(A^{\beta / 2} B^{\beta} A^{\beta / 2}\right) .
$$


Substituting $A$ for $A^{1 / 2}$ in (6.7) and let $0<\alpha<\beta=1$. Then

$$
\log \lambda^{1 / \alpha}\left(A^{\alpha} B^{\alpha} A^{\alpha}\right) \prec \log \lambda(A B A) \Leftrightarrow \log \lambda\left(A^{\alpha} B^{\alpha} A^{\alpha}\right) \prec \log \lambda(A B A)^{\alpha} .
$$

6.7 Lemma. The inequality (6.1) is equivalent to inequality

where $\hat{Y}=\left(X^{-1 / 2 \delta} Y^{1 / \delta} X^{-1 / 2 \delta}\right)^{\delta}$.

$$
\forall A>0: \operatorname{det}\left[A^{\delta}\left(A^{-1} \hat{Y}^{1 / \delta} A^{-1}\right)^{\delta} A^{\delta}-I_{n}\right] \geq \operatorname{det}\left[\hat{Y}-I_{n}\right],
$$

Proof. Let $Q_{0}=X^{1 / 2 \delta}$. We can write a matrix $Q \in G L(n, \mathbb{C})$ in the form $Q=Q_{0} \cdot A \cdot U$, where $A>0, U \in U(n)$. Let $\hat{Y}=\varphi_{Q_{0}}^{-1}(Y)=\left(X^{-1 / 2 \delta} Y^{1 / \delta} X^{-1 / 2 \delta}\right)^{\delta}$. Then

$$
\operatorname{det}(Y \stackrel{Q}{\ominus} X)=\operatorname{det} \varphi_{Q_{0} A U}\left[\varphi_{Q_{0} A U}^{-1}(Y)-\varphi_{Q_{0} A U}^{-1}(X)\right]=\left|\operatorname{det} Q_{0}\right|^{2 \delta} \operatorname{det}\left[A^{\delta}\left(A^{-1} \hat{Y}^{1 / \delta} A^{-1}\right)^{\delta} A^{\delta}-I_{n}\right] .
$$

Similarly, for the matrix $Y \stackrel{\tau_{28}^{-1}(X)}{\ominus} X$ we obtain

$$
\operatorname{det}\left(Y \stackrel{\tau_{2 \delta}^{-1}(X)}{\ominus} X\right)=\operatorname{det}\left(Y \stackrel{Q_{0}}{\ominus} X\right)=\left|\operatorname{det} Q_{0}\right|^{2 \delta} \operatorname{det}\left[\varphi_{Q_{0}}^{-1}(Y)-\varphi_{Q_{0}}^{-1}(X)\right]=\left|\operatorname{det} Q_{0}\right|^{2 \delta} \operatorname{det}\left[\hat{Y}-I_{n}\right] .
$$

Thus, inequality (6.1) is equivalent to inequality (6.8).

6.8 Lemma. Let $0<\delta \leq 1$, A $>0$. If $Y \geq I_{n}$, then $A^{\delta}\left(A^{-1} Y^{1 / \delta} A^{-1}\right)^{\delta} A^{\delta} \geq I_{n}$.

Proof. If $Y \geq I_{n}$, then $Y^{1 / \delta} \geq I_{n}$. We obtain $A^{-1} Y^{1 / \delta} A^{-1} \geq A^{-2}$. Since $0<\delta \leq 1$, then $f(X)=X^{\delta}$ is monotone on $\mathbb{P}_{n}$. Then $\left(A^{-1} \tilde{Y}^{1 / \delta} A^{-1}\right)^{\delta} \geq A^{-2 \delta}$, i.e. $A^{\delta}\left(A^{-1} \hat{Y}^{1 / \delta} A^{-1}\right)^{\delta} A^{\delta} \geq I_{n}$.

6.9 Proof of Theorem 6.1. Let $X, Y \in \mathbb{P}_{n}^{+}$and $X \leq_{\delta} Y$. Taking into account Theorem 4.6, we obtain $\quad X^{1 / \delta} \leq Y^{1 / \delta}$, i.e. $\hat{Y}=\left(X^{-1 / 2 \delta} Y^{1 / \delta} X^{-1 / 2 \delta}\right)^{\delta} \geq I_{n}$. Using Lemma 6.8, we get $A^{\delta}\left(A^{-1} \hat{Y}^{1 / \delta} A^{-1}\right)^{\delta} A^{\delta} \geq I_{n}$. The eigenvalues of $C=A^{\delta}\left(A^{-1} \hat{Y}^{1 / \delta} A^{-1}\right)^{\delta} A^{\delta}$ and $\hat{Y}$ are denoted by $\lambda(C)=\left(\lambda_{1}, \lambda_{2}, \cdots, \lambda_{n}\right)_{\downarrow} \in \mathbb{D}_{+}^{n}(1)$ and $\mu(\hat{Y})=\left(\mu_{1}, \mu_{2}, \cdots, \mu_{n}\right)_{\downarrow} \in \mathbb{D}_{+}^{n}(1)$. Let $B=A^{-1} \hat{Y}^{1 / \delta} A^{-1}$. By Lemma 6.6, $\log \lambda\left(A^{\delta} B^{\delta} A^{\delta}\right) \prec \log \lambda(A B A)^{\delta}$ so that

$$
\log \lambda\left[A^{\delta}\left(A^{-1} \hat{Y}^{1 / \delta} A^{-1}\right)^{\delta} A^{\delta}\right] \prec \log \lambda\left[A\left(A^{-1} \hat{Y}^{1 / \delta} A^{-1}\right) A\right]^{\delta}=\log \mu(\hat{Y}) .
$$

Using Proposition 6.3, we get

$$
\operatorname{det}\left[A^{\delta}\left(A^{-1} \hat{Y}^{1 / \delta} A^{-1}\right)^{\delta} A^{\delta}-I_{n}\right]=\left(\lambda_{1}-1\right) \cdots\left(\lambda_{n}-1\right) \geq\left(\mu_{1}-1\right) \cdots\left(\mu_{n}-1\right)=\operatorname{det}\left[\hat{Y}-I_{n}\right],
$$

which, according to Lemma 6.7, is equivalent to inequality (6.1).

The condition $X \leq_{\delta} Y$ can be omitted for $2 \times 2$ matrices. Indeed, let $B=A^{-1} \hat{Y}^{1 / \delta} A^{-1}$, $\hat{Y}=\left(X^{-1 / 2 \delta} Y^{1 / \delta} X^{-1 / 2 \delta}\right)^{\delta}$ are $2 \times 2$ matrices. The eigenvalues of $C=A^{\delta}\left(A^{-1} \hat{Y}^{1 / \delta} A^{-1}\right)^{\delta} A^{\delta}$ and $\hat{Y}$ are denoted by $\lambda(C)=\left(\lambda_{1}, \lambda_{2}\right)$ and $\mu(\hat{Y})=\left(\mu_{1}, \mu_{2}\right)$. Then

Using Theorem 6.5, we get

$$
\lambda_{1} \lambda_{2}=\operatorname{det}\left[A^{\delta}\left(A^{-1} \hat{Y}^{1 / \delta} A^{-1}\right)^{\delta} A^{\delta}\right]=\operatorname{det} \hat{Y}=\mu_{1} \mu_{2} .
$$

$$
\lambda_{1}+\lambda_{2}=\operatorname{tr}\left(A^{\delta} \cdot\left(A^{-1} \hat{Y}^{1 / \delta} A^{-1}\right)^{\delta} \cdot A^{\delta}\right) \leq \operatorname{tr}\left(A \cdot A^{-1} \hat{Y}^{1 / \delta} A^{-1} \cdot A\right)^{\delta}=\operatorname{tr} \hat{Y}=\mu_{1}+\mu_{2} .
$$

Combining (6.9) and (6.10), we obtain

$$
\operatorname{det}\left[A^{\delta}\left(A^{-1} \hat{Y}^{1 / \delta} A^{-1}\right)^{\delta} A^{\delta}-I_{2}\right]=\lambda_{1} \lambda_{2}-\left(\lambda_{1}+\lambda_{2}\right)+1 \geq \mu_{1} \mu_{2}-\left(\mu_{1}+\mu_{2}\right)+1=\operatorname{det}\left[\hat{Y}-I_{2}\right] .
$$

In conclusion, we prove for vector multispace $\mathbb{H}_{\delta}(n)$ the Minkowski's determinant inequality. 
6.10 Theorem (Minkowski's determinant inequality). Let $\delta>0$ and $X, Y \in \mathbb{H}_{\delta}(n)$ are positive definite $n \times n$-matrices. Then

$$
[\operatorname{det}(Y \stackrel{Q}{\oplus} X)]^{1 / n} \geq[\operatorname{det} Y]^{1 / n}+[\operatorname{det} X]^{1 / n}, \quad \forall Q \in G L(n, \mathbb{C})
$$

with equality if and only if $Y=c X$ for some $c>0$.

Proof. By Corollary 3.7, we can assume that $Q \in S L(n, \mathbb{C})$. Then $\operatorname{det} \varphi_{Q}(X)=\operatorname{det} X$ and we obtains

$$
\begin{aligned}
{[\operatorname{det}(Y \stackrel{Q}{\oplus} X)]^{1 / n}=\left[\operatorname{det} \varphi_{Q}\left[\varphi_{Q}^{-1}(Y)+\varphi_{Q}^{-1}(X)\right]\right]^{1 / n}=\left[\operatorname{det}\left[\varphi_{Q}^{-1}(Y)+\varphi_{Q}^{-1}(X)\right]\right]^{1 / n} \geq } \\
\geq\left[\operatorname{det} \varphi_{Q}^{-1}(Y)\right]^{1 / n}+\left[\operatorname{det} \varphi_{Q}^{-1}(X)\right]^{1 / n}=(\operatorname{det} Y)^{1 / n}+(\operatorname{det} X)^{1 / n},
\end{aligned}
$$

where for ordinary addition the well-known (see [11], p. 510) Minkowski inequality is used. For the case of equality in (6.11) we have $\left[\operatorname{det}\left[\varphi_{Q}^{-1}(Y)+\varphi_{Q}^{-1}(X)\right]\right]^{1 / n}=\left[\operatorname{det} \varphi_{Q}^{-1}(Y)\right]^{1 / n}+\left[\operatorname{det} \varphi_{Q}^{-1}(X)\right]^{1 / n}$. Then $\varphi_{Q}^{-1}(Y)=c \varphi_{Q}^{-1}(X)$, i.e. $Y=c X$ for some $c>0$.

\section{Analog of an indefinite metric on the set of positive definite matrices}

By Theorem 3.8 and Proposition 3.4(iv), each addition operation in a multispace $\mathbb{H}_{\delta}(n)$ is induced by some positive definite matrix $A>0$. On the set $\mathbb{P}_{n}^{+} \subset \mathbb{H}_{\delta}(n)$ of positive definite matrices we introduce a ternary operation $\{X, A, Y\} \mapsto Z$ by the relation $Z=X \stackrel{\tau_{2 \delta}^{-1}(A)}{\oplus} Y$.

7.1 Proposition. Operation $Z=X \stackrel{\tau_{2 \delta}^{-1}(A)}{\oplus} Y$ retains its form under all the transformations $X^{\prime}=\varphi_{Q}(X), \varphi_{Q} \in G_{\delta}(n)$ in $\mathbb{P}_{n}^{+}$, i.e.

$$
\varphi_{Q}\left(X \stackrel{\tau_{2 \delta}^{-1}(A)}{\oplus} Y\right)=X^{\prime} \stackrel{\tau_{2 \delta}^{-1}\left(A^{\prime}\right)}{\oplus} Y^{\prime}
$$

Proof. By Propositions 3.4 (i), 3.6 we have

$$
\varphi_{Q}\left(X \stackrel{\tau_{2 \delta}^{-1}(A)}{\oplus} Y\right)=\varphi_{Q}(X) \stackrel{Q \cdot \tau_{2 \delta}^{-1}(A)}{\oplus} \varphi_{Q}(Y)=X^{\prime} \stackrel{\left|Q \cdot \tau_{2 \delta}^{-1}(A)\right|_{L}}{\oplus} Y^{\prime},
$$

where $\left|Q \cdot \tau_{2 \delta}^{-1}(A)\right|_{L}=\left(Q \cdot A^{1 / \delta} \cdot Q^{*}\right)^{1 / 2}=\left[\left(Q \cdot A^{1 / \delta} \cdot Q^{*}\right)^{\delta}\right]^{1 / 2 \delta}=\tau_{2 \delta}^{-1}\left[\varphi_{Q}(A)\right]=\tau_{2 \delta}^{-1}\left(A^{\prime}\right)$

7.2 Proposition. Let $X, Y \in \mathbb{H}_{\delta}(n)$ be positive definite matrices and $\delta>0$. Then

$$
Y \stackrel{\tau_{2 \delta}^{-1}(X)}{\ominus} X=Y \stackrel{\tau_{2 \delta}^{-1}(Y)}{\ominus} X
$$

Proof. It is sufficient to show that for some $\varphi_{Q} \in S G_{\delta}(n)$ the equality $\varphi_{Q}\left(Y \stackrel{\tau_{2 \delta}^{-1}(X)}{\ominus} X\right)=\varphi_{Q}\left(Y \stackrel{\tau_{2 \delta}^{-1}(Y)}{\ominus} X\right)$ is satisfied. By Lemma 4.7 there exists a transformation $\varphi_{Q} \in S G_{\delta}(n)$ such that $\varphi_{Q}(X)=\lambda_{0} I_{n}$, $\varphi_{Q}(Y)=\Lambda_{\mu}=\operatorname{diag}\left\{\mu_{1}, \cdots, \mu_{n}\right\}$. Using Proposition 7.1 we have

$$
\varphi_{Q}\left(Y \stackrel{\tau_{2 \delta}^{-1}(X)}{\ominus} X\right)=\Lambda_{\mu} \stackrel{\tau_{2 \delta}^{-1}\left(\lambda_{0} I_{n}\right)}{\ominus} \lambda_{0} I_{n} ; \quad \varphi_{Q}\left(Y \stackrel{\tau_{2 \delta}^{-1}(Y)}{\ominus} X\right)=\Lambda_{\mu} \stackrel{\tau_{2 \delta}^{-1}\left(\Lambda_{\mu}\right)}{\ominus} \lambda_{0} I_{n} .
$$

Since for each matrix $\Lambda_{0}=\operatorname{diag}\left\{\alpha_{1}, \cdots, \alpha_{n}\right\} \in G L(n, \mathbb{C})$ the equality $\Lambda_{\mu} \ominus \stackrel{\Lambda}{0}_{0} \lambda_{0} \cdot I_{n}=\Lambda_{\mu}-\lambda_{0} \cdot I_{n}$ is satisfied, then $\varphi_{Q}\left(Y \stackrel{\tau_{2 \delta}^{-1}(X)}{\ominus} X\right)=\Lambda_{\mu} \stackrel{\tau_{2 \delta}^{-1}\left(\lambda_{0} I_{n}\right)}{\ominus} \lambda_{0} I_{n}=\Lambda_{\mu}-\lambda_{0} I_{n}=Y \stackrel{\tau_{2 \delta}^{-1}\left(\Lambda_{\mu}\right)}{\ominus} X=\varphi_{Q}\left(Y \stackrel{\tau_{2 \delta}^{-1}(Y)}{\ominus} X\right)$..

7.3 Definition. Let $X \leq_{\delta} Y$ be positive definite matrices of the ordered vector multispace $\mathbb{H}_{\delta}(n)$, $0<\delta \leq 1$. The number $\Delta \sigma(X, Y)=\left[\operatorname{det}\left(Y \stackrel{\tau_{2 \delta}^{-1}(Y)}{\ominus} X\right)\right]^{1 / n}$ will be called the interval from point $X$ to point $Y$. 
7.4 Theorem (Triangle Reverse Inequality). Let $X, Y, Z$ be positive defined matrices of the ordered vector multispace $\mathbb{H}_{\delta}(n), 0<\delta \leq 1$. If $X \leq_{\delta} Y \leq_{\delta} Z$, then

$$
\Delta \sigma(X, Z) \geq \Delta \sigma(X, Y)+\Delta \sigma(Y, Z)
$$

with equality if and only if $\left(Z \stackrel{\tau_{2 \delta}^{-1}(X)}{\ominus} X\right)=c \cdot\left(Y \stackrel{\tau_{2 \delta}^{-1}(X)}{\ominus} X\right)$ for some $c>0$.

Proof. Using Minkowski’s determinant inequality and Theorem 6.1 we obtain

$$
\begin{aligned}
& \left.\Delta \sigma(X, Z)=\left[\operatorname{det}\left(Z \stackrel{\tau_{2 \delta}^{-1}(X)}{\ominus} X\right)\right]^{1 / n}=\left[\operatorname{det}\left[\left(Z \stackrel{\tau_{2 \delta}^{-\frac{1}{2}}(X)}{\ominus} Y\right) \stackrel{\tau_{2 \delta}^{-1}(X)}{\oplus}\left(Y \stackrel{\tau_{2 \delta}^{-1}(X)}{\ominus} X\right)\right]\right]\right]^{1 / n} \geq[(6,11)] \geq\left[\operatorname{det}\left(Y \stackrel{\tau_{2 \delta}^{-1}(X)}{\ominus} X\right)\right]^{1 / n}+ \\
& \quad+\left[\operatorname{det}\left(Z \stackrel{\tau_{2 \delta}^{-1}(X)}{\ominus} Y\right)\right]^{1 / n} \geq[(6,1)] \geq\left[\operatorname{det}\left(Y \stackrel{\tau_{2 \delta}^{-1}(X)}{\ominus} X\right)\right]^{1 / n}+\left[\operatorname{det}\left(Z \stackrel{\tau_{2 \delta}^{-1}(Y)}{\ominus} Y\right)\right]^{1 / n}=\Delta \sigma(X, Y)+\Delta \sigma(Y, Z) .
\end{aligned}
$$

The equality $\Delta \sigma(X, Z)=\Delta \sigma(X, Y)+\Delta \sigma(Y, Z)$ is true if only if

$$
\left.\left[\operatorname{det}\left[\left(Y \stackrel{\tau_{2 \delta}^{-1}(X)}{\ominus} X\right)\right] \stackrel{\tau_{2 \delta}^{-1}(X)}{\oplus}\left(Z \stackrel{\tau_{2 \delta}^{-1}(X)}{\ominus} Y\right)\right]\right]^{1 / n}=\left[\operatorname{det}\left(Y \stackrel{\tau_{2 \delta}^{-1}(X)}{\ominus} X\right)\right]^{1 / n}+\left[\operatorname{det}\left(Z \stackrel{\tau_{2 \delta}^{-1}(X)}{\ominus} Y\right)\right]^{1 / n} .
$$

By Theorem 6.10 we have $\left(Z \stackrel{\tau_{2 \delta}^{-1}(X)}{\ominus} Y\right)=a \cdot\left(Y \stackrel{\tau_{2 \delta}^{-1}(X)}{\ominus} X\right)$ for some $a>0$. From here we get $\left(Z \stackrel{\tau_{2 \delta}^{-1}(X)}{\ominus} X\right)=c \cdot\left(Y \stackrel{\tau_{2 \delta}^{-1}(X)}{\ominus} X\right)$ for some $c>0$.

7.5 Corollary. Let $X_{0}, X_{1}$ be positive defined matrices of the ordered vector multispace $\mathbb{H}_{\delta}(n)$, $0<\delta \leq 1$. If $X_{0} \leq_{\delta} X_{1}$, then there exists a unique chain of "maximum length" connecting points $X_{0}, X_{1}$. This maximum chain has a parametrization

$$
X(s)=(1-s) \cdot X_{0} \stackrel{\tau_{2 s}^{-1}\left(X_{0}\right)}{\oplus} s \cdot X_{1}, \quad 0 \leq s \leq 1,
$$

which is natural in the sense that $\Delta \sigma\left(X_{0}, X(s)\right)=s \cdot \Delta \sigma\left(X_{0}, X_{1}\right)$ for each $0 \leq s \leq 1$.

Proof. The maximum length is a consequence of Triangle Reverse Inequality. The interval from point $X_{0}$ to point $X(s)$ is

$$
\Delta \sigma\left(X_{0}, X(s)\right)=\left[\operatorname{det}\left(X(s) \stackrel{\tau_{2 \delta}^{-1}\left(X_{0}\right)}{\ominus} X_{0}\right)\right]^{1 / n}=\left[\operatorname{det}\left(s\left(X_{1} \stackrel{\tau_{2 \delta}^{-1}\left(X_{0}\right)}{\ominus} X_{0}\right)\right)\right]^{1 / n}=s \cdot \Delta \sigma\left(X_{0}, X_{1}\right) . \boldsymbol{\square} .
$$

7.6 Example (Causal Lorentz manifold of dimension 4). Let $X_{0}, Y$ be positive defined $2 \times 2$ matrices and $0<\delta \leq 1$. Each Hermitian $2 \times 2$ matrix $A$ uniquely represented in the form

$$
A=\left(\begin{array}{cc}
c t+z & x+i y \\
x-i y & c t-z
\end{array}\right)
$$

with fixed constant $c>0$. Hermitian matrix

$$
Y \stackrel{\tau_{2 \delta}^{-1}\left(X_{0}\right)}{\ominus} X_{0}=\left(\begin{array}{cc}
c \cdot \tilde{\Delta} t+\tilde{\Delta} z & \tilde{\Delta} x+i \tilde{\Delta} y \\
\tilde{\Delta} x-i \tilde{\Delta} y & c \cdot \tilde{\Delta} t-\tilde{\Delta} z
\end{array}\right)
$$

is an element of the tangent space at the point $X_{0}$. Here the representation (7.5) for $Y \stackrel{\tau_{2 \delta}^{-1}\left(X_{0}\right)}{\ominus} X_{0}$ defines each of numbers $\tilde{\Delta} t, \tilde{\Delta} x, \tilde{\Delta} y, \tilde{\Delta} z$. Then

$$
\operatorname{det}\left(Y \stackrel{\tau_{2 \delta}^{-1}\left(X_{0}\right)}{\ominus} X_{0}\right)=c^{2} \cdot \tilde{\Delta} t^{2}-\tilde{\Delta} x^{2}-\tilde{\Delta} y^{2}-\tilde{\Delta} z^{2}
$$

is the invariant of the group $S G_{\delta}(2)$. This invariant defines a indefinite metric on the manifold of positive definite $2 \times 2$ matrices at point $X_{0}$. The partial order relation $X \leq_{\delta} Y$ is a model of causal relationship between "events" (7.5). Positive definite matrices model "observed" events. Parameter $\tau=s \cdot \Delta \sigma\left(X_{0}, X_{1}\right)$ (Definition 7.3) can be considered as the free particle's own time. 
The group $S G_{\delta}(2)$ of nonlinear transformations of multispace $\mathbb{H}_{\delta}(2)$ is a representation of the arcwise connected component of the unit of Lorenz group. Group $S G_{\delta}(2)$, unlike the Lorentz group, is defined not by one, but by two parameters $c>0$ and $0<\delta \leq 1$. Note that at $c \rightarrow \infty$ and each $0<\delta \leq 1$ the nonlinear transformations of the group $S G_{\delta}(2)$ are transform into linear Galilean transformations.

\section{REFERENCES}

[1]. S. Honmaa,T. Nogawa, Isometries of the geodesic distances for the space of invertible positive operators and matrices, Linear Algebra Appl., 444 (2014) 152-164.

[2]. O. Hatori, L. Molnar, Isometries of the unitary groups and Thompson isometries of the spaces of invertible positive elements in $C^{*}$-algebras, J. Math. Anal. Appl. 409 (2014) 158 167.

[3]. F. Hiai, D. Petz, Riemannian metrics on positive definite matrices related to means, Linear Algebra Appl., 430 (2009) 3105-3130.

[4]. M. Ito, Y. Seo, T. Yamazaki and M. Yanagida, On a geometric property of positive definite matrices cone, Banach J. Math. Anal. 3 (2009), no. 2, 64-76.

[5]. F. Hansen, An operator inequality, Mathematische Annalen 246 (1980), 249-250.

[6]. H. Araki, “On an inequality of Lieb and Thirring”, Lett. Math. Phys. 19(1990), pp. 167-170.

[7]. E. Lieb and W. Thirring, in Studies in Mathematical Physics (Eds. E. Lieb, B. Simon and A.Wightman), pp. 301-302, Princeton Press, 1976.

[8]. B.-Y. Wang and M.-P. Gong, Some eigenvalue inequalities for positive semidefinite matrix power products, Linear Algebra Appl., 184(1993), pp. 249-260.

[9]. B-Y. Wang and F. Zhang, "Trace and Eigenvalue Inequalities for Ordinary and Hadamard Products of Positive Semidefinite Hermitian Matrices", SIAM J. Matrix Anal. Appl.16(1995), pp. 1173-1183.

[10]. A. W. Marshall and I. Olkin, Inequalities: Theory of majorization and its applications, Academic Press, San Diego, CA, 1979.

[11]. R.A. Horn and C.R. Johnson, Matrix Analysis, Cambridge University Press, 1985.

[12]. R. Bhatia, Matrix Analysis, Springer, 1997.

[13]. R. Bhatia, Positive Definite Matrices, Princeton University Press, 2007.

[14]. H. Schaefer, Topological Vector Spaces, New York, London, 1966. 